\title{
EFECTO DE LA VARIEDAD Y LA FERTILIZACIÓN EN INDICADORES DE CALIDAD PROTEICA IN-VITRO DE DOS VARIEDADES Y UNA LÍNEA DE GARBANZO (Cicer arietinum)
}

\author{
VARIETY AND FERTILIZATION EFFECT ON \\ INDICATORS OF PROTEIN QUALITY IN-VITRO OF TWO \\ VARIETIES AND ONE LINE OF CHICKPEA (Cicer arietinum)
}

\author{
Alma Guadalupe Cota G., Grelda Acela Yañez F., Elsa Julia Esquer M., \\ Rosario Anduaga C., Jesús Manuel Barrón H.
}

Departamento de Investigación y Posgrado en Alimentos, Universidad de Sonora, México.

\begin{abstract}
Protein quality in relation to the degree of fertilization of two varieties (Costa and Blanco Sinaloa) and an advanced line (Hoga L10) of chickpeas obtained from the Campo Experimental Costa de Hermosillo, Sonora, Mexico from INFAP was evaluated. They were grown in similar conditions with 3 levels of nitrogen fertilization (60, 120 and 200K/Ha.). Protein and non-protein nitrogen, digestibility and available lysine were analyzed. Significant differences between the varieties and the advanced line in protein and non-protein nitrogen and available lysine were found. The effect of the degree offertilization on protein quality was significant, showing that a level of 120K/ Ha an increase is observed in protein and non-protein nitrogen and in the percent of digestibility. No significant differences were observed between 60 and 200K/Ha. Available lysine was higher at the 200K/Ha level. These indicators of protein quality can be used to complement commercial and agricultural aspects that are evaluated for improvement and selection of new varieties of chickpeas.
\end{abstract}

Palabras clave: protein quality in-vitro; chickpea; varieties ; fertilization; nitrogen.

Este trabajo fue recibido el 17 de Marzo de 2009 y aceptado para ser publicado el 9 de Abril de 2010.

\section{INTRODUCCIÓN}

El garbanzo es una leguminosa ampliamente consumida en todo el mundo (1), y se le considera una buena fuente de carbohidratos y proteínas de fácil adquisición y bajo costo. Cuando esta leguminosa se combina con cereales en proporciones adecuadas da como resultado una proteína con un mejor perfil de aminoácidos $(2,3)$.

A nivel mundial México ocupa el segundo lugar como exportador de garbanzo y el tercero en producción. El área sembrada en el año 2006 fue de 118,490 Ha. con una producción de 162,382 toneladas (4), la que fue destinada al mercado internacional por su alta calidad.

En México se encuentra el Instituto Nacional de Investigaciones Forestales, Agrícolas y Pecuarias (INIFAP) en donde al igual que en otros países, continuamente se está trabajando en el mejoramiento genético de garbanzo Kabuli. Esto, con la finalidad de obtener nuevas variedades con mejor calidad de exportación, alto rendimiento, facilidad a la cosecha mecánica y menor riesgo al daño de heladas y enfermedades $(5,6)$.

Una de las etapas del mejoramiento genético consiste en sembrar las líneas avanzadas seleccionadas en diferentes suelos y prácticas de cultivo como lo es la fertilización, con la finalidad de validar las cualidades agronómicas encontradas, para posteriormente culminar en la liberación de la nueva variedad (7).

Es importante mencionar que el mejoramiento del garbanzo se basa únicamente en criterios agronómicos y siendo ésta una leguminosa que se complementa con los cereales para la obtención de un mejor balance de aminoácidos esenciales, se considera de gran importancia brindar información en el aspecto nutricional. Esta 
información complementa los resultados agronómicos generados por los fitomejoradores, incrementando los criterios de selección y contribuyendo a una elección de variedades mejoradas no solo agronómicamente sino también nutricionalmente, haciéndolas de esta manera más aptas para la competencia comercial. Es por lo anterior que en este trabajo se planteó estudiar la calidad proteica in vitro de dos variedades y una línea avanzada de garbanzo sembradas con diferentes niveles de fertilización.

\section{MATERIALES Y MÉTODO Muestras de garbanzo}

Se utilizaron dos variedades comerciales de garbanzo: Costa y Blanco Sinaloa y una línea avanzada Hoga L10, las cuáles fueron sembradas con tres niveles de fertilización de Nitrógeno: 60, 120 y 200K/Ha. Estas muestras fueron obtenidas del Campo Experimental de la Costa de Hermosillo, Sonora, México dependiente de INIFAP. Las muestras se limpiaron mecánica y manualmente empacándose en bolsas de papel y polietileno; se almacenaron en refrigeración a $4^{\circ} \mathrm{C}$ para su posterior análisis.

\section{Composición química}

Las muestras fueron analizadas en su contenido de humedad, cenizas, proteína, grasa y fibra cruda de acuerdo a los métodos oficiales (44-15, 08-01,46-13, 30-10 y 32-10) de la AACC (8).

\section{Nitrógeno proteico y no proteico}

El contenido de nitrógeno no proteico (NNP) fue determinado mediante extracción directa utilizando ácido tricloroacético (TCA) al 10\%, basado en el método propuesto por Singh y Jambunathan (9).

\section{Digestibilidad in vitro}

El método utilizado para la determinación de digestibilidad in vitro fue el propuesto por Saterlee et al (10). Está determinación se basa en un sistema de 4 enzimas, las cuales se ponen en contacto con una solución acuosa que contenga $10 \mathrm{mg}$ de nitrógeno provenientes de la muestra a probar, utilizando caseinato de sodio como proteína control.

En este método se utilizan dos soluciones enzimáticas: Solución A (Tripsina, quimotripsina y peptidasa, Sigma) y Solución B (proteasa bacterial, Sigma) que fueron ajustadas a un PH $8.00 \pm 0.05$. Un mililitro de solución A se adiciona a la solución de la muestra y es mantenida a $37^{\circ} \mathrm{C}$ por $10 \mathrm{~min}$. Posteriormente se adiciona la solución $\mathrm{B}$ a la reacción y la temperatura se cambia a $55^{\circ} \mathrm{C}$ por $9 \mathrm{~min}$. Después de este tiempo la temperatura es regresada a $37^{\circ} \mathrm{C}$ por $1 \mathrm{~min}$. El pH es registrado a los 20 minutos del ensayo. El valor de $\mathrm{pH}$ es utilizado en el cálculo del \% de digestibilidad. Todas las muestras fueron analizadas por triplicado y los resultados fueron calculados por la ecuación:

$\%$ de Digestibilidad $=234.84-22.56(\mathrm{X})$

Donde $\mathrm{X}$ es el $\mathrm{pH}$ a los 20 minutos

\section{Lisina disponible}

Para llevar a cabo esta determinación se utilizó el método propuesto por Hurrel et al (11). Descrito como Dye Binding Capacity (DBC). Se realizó una curva estándar usando concentraciones conocidas de colorante naranja 12 relacionando la absorbancia a $475 \mathrm{~nm}$. Las muestras fueron analizadas por triplicado y los resultados fueron expresados en $\mathrm{g}$ de lisina/16 g de nitrógeno de la muestra.

\section{Diseño y análisis estadístico}

El diseño de experimentos utilizado en este estudio fue un factorial de $3 \times 3$, donde los factores de estudio fueron las variedades (Costa, Blanco Sinaloa y la línea avanzada Hoga L10), y las dosis de fertilización (60, 120 y $200 \mathrm{Kg}$ de N/Ha).

Los datos fueron analizados estadísticamente por un ANDEVA y prueba de comparación de medias de Tukey $(\mathrm{p}<0.05)$ utilizando el programa estadístico JMP 6.0 para Windows (12).

\section{RESULTADOS Y DISCUSIÓN Composición química}

El efecto de la variedad fue observado en todas los componentes, la línea Hoga L10 fue la que presentó mayor contenido de grasa, en cenizas la variedad Costa mostró el mayor contenido, y el porcentaje de proteína y fibra fueron mayores en la variedad Blanco Sinaloa (tabla 1). Es claro que el factor genético tiene un efecto importante en la composición química, sin embargo sumado a la variedad otros factores tales como el tipo de suelo, condiciones de fertilización, preparación del terreno y fecha de siembra, entre otros, pueden contribuir a estos resultados.

A excepción de grasa, la influencia del grado de fertilización fue encontrada en la composición química. En las dos variedades Costa y Blanco Sinaloa los valores más altos de cenizas fueron los obtenidos de las fertilizaciones de $120 \mathrm{~K} / \mathrm{Ha}$. Mientras que para la línea avanzada Hoga L10 el valor más alto fue para la fertilización con nivel $60 \mathrm{~K} / \mathrm{Ha}$. Con respecto a proteína a excepción de la línea Hoga L10 las variedades de garbanzo que recibieron una fertilización de 120K/Ha., presentaron un mayor porcentaje de proteína, y el más bajo fue para los granos 
de garbanzo que estuvieron expuestos a una fertilización de Nitrógeno de $200 \mathrm{~K} / \mathrm{Ha}$. Las muestras de garbanzo, que recibieron dosis de $200 \mathrm{Kg} / \mathrm{Ha}$ presentaron un mayor contenido de fibra cruda y carbohidratos. Se observa además la influencia de la interacción de fertilización y variedad, la cual es más evidente en el contenido de cenizas debido a que en las tres muestras de garbanzo se presentan diferencias significativas entre las dosis de nitrógeno usadas. En el caso de proteína, fibra cruda y carbohidratos las diferencias son presentadas solo en Blanco Sinaloa.

El efecto del factor genético así como de la localidad en ciertos componentes químicos del garbanzo y otras leguminosas ha sido reportado por Vargas-Torres et al (13) quienes encontraron diferencias en el contenido de cenizas en frijol; de igual manera Pérez et al (14) y Ravi y Harte (6) encontraron variabilidad en el contenido de proteína en variedades de garbanzo.

\section{Efecto del grado de fertilización y variedad en el contenido de nitrógeno}

Nitrógeno no proteico. Se observó que la variedad Blanco Sinaloa presenta los valores mayores de nitrógeno proteico, seguido de Costa y Hoga L10, indicando de esta forma el efecto de la variedad (figura 1). En relación a la fertilización en la misma figura se observa que cada variedad presentó un comportamiento diferente. La variedad Hoga L10 al nivel de fertilización de 120K/Ha obtuvo una menor cantidad de nitrógeno no proteico, mientras que para las variedades Costa y Blanco Sinaloa con fertilizaciones 200 y $60 \mathrm{~K} / \mathrm{Ha}$ respectivamente fueron las que mostraron los valores más bajos. Estás diferencias fueron estadísticamente significativas ( $\mathrm{p}<0.05)$.

Los valores de NNP encontrados en esta investigación estuvieron dentro de los rangos reportados en otros estudios: Navarro (15) obtuvo valores de NNP desde $0.143 \%$ hasta $0.518 \%$, en variedades de garbanzo y Singh y Jambunathan (9) quienes estudiaron 98 líneas de garbanzo reportaron valores entre 0.16 y $0.73 \%$ de nitrógeno no proteico, encontrando además una correlación positiva con el nitrógeno total.

Nitrógeno proteico. Se presentaron variaciones significativas $(\mathrm{P}<0.05)$ entre las muestras de garbanzo analizadas siendo la variedad Blanco Sinaloa la que presentó un mayor contenido (figura 2). En esta misma figura se observó que el contenido de nitrógeno proteico se ve afectado significativamente por el grado de fertilización. La línea avanzada Hoga L10 en los tres niveles de fertilización no presentó diferencias significativas $(\mathrm{p}<0.05)$. En la variedad Costa el nivel de fertilización

\section{TABLA 1}

Composición química de variedades de garbanzo con diferentes grados de fertilización ${ }^{1,2}$

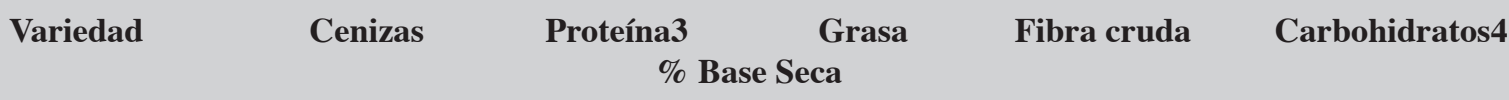

Hoga L-10

60

120

200

Costa

60

120

200 $22.10^{\mathrm{abc}}$

$20.87^{c}$

$21.25^{\mathrm{bc}}$

$21.45^{\mathrm{abc}}$

3.38

$22.66^{\text {abc }}$

$3.28^{\mathrm{d}}$

$3.12^{\mathrm{e}}$

$3.47^{\mathrm{bc}}$

$3.37^{\mathrm{cd}}$ $23.56^{\mathrm{ab}}$

$23.81^{\mathrm{a}}$

$20.11^{c}$ $6.97^{\mathrm{a}}$

$6.10^{\mathrm{abc}}$

$6.71^{\mathrm{ab}}$

$5.88^{\mathrm{bc}}$

$5.81^{\mathrm{bc}}$

$5.86^{\text {bc }}$ $1.03^{\mathrm{c}}$

$1.37^{\mathrm{bc}}$

$1.53^{\mathrm{abc}}$

$1.70^{\mathrm{ab}}$

$1.43^{\mathrm{abc}}$

$1.75^{\mathrm{ab}}$

$1.68^{\mathrm{ab}}$

$1.33^{\text {bc }}$

$1.92^{\mathrm{a}}$ $66.39^{\mathrm{bc}}$

$68.21^{\text {abc }}$

$67.36^{\mathrm{abc}}$
60

200 $5.68^{c}$

$5.78^{\mathrm{bc}}$

$5.47^{\mathrm{c}}$ $67.59^{\mathrm{abc}}$

$66.18^{\text {abc }}$

$68.94^{\mathrm{ab}}$

${ }^{1}$ Valores con diferente letra son significativamente diferentes $(\mathrm{p}<0.05)$

${ }^{2}$ Los resultados son promedio de triplicados.

${ }^{3}$ Proteína total 6.25 (Factor de conversión).

${ }^{4}$ Carbohidratos obtenidos por diferencia. 
de $120 \mathrm{~K} / \mathrm{Ha}$ fue el que mostró los valores mas altos de NP, seguido de los niveles de 60 y $200 \mathrm{~K} / \mathrm{Ha}$ La variedad Blanco Sinaloa con nivel de 120 presentó los valores mayores de NP, encontrándose diferencias significativas $(\mathrm{p}<0.05)$ con niveles de fertilización de 120 y $200 \mathrm{~K} /$ Ha. A excepción de Hoga L10 se puede decir que las variedades con 120 de fertilización mostraron los valores más altos de nitrógeno proteico. Las variedades con

\section{FIGURA 1}

Nitrógeno no proteico presente en variedades de garbanzo sembradas a diferentes niveles de fertilización

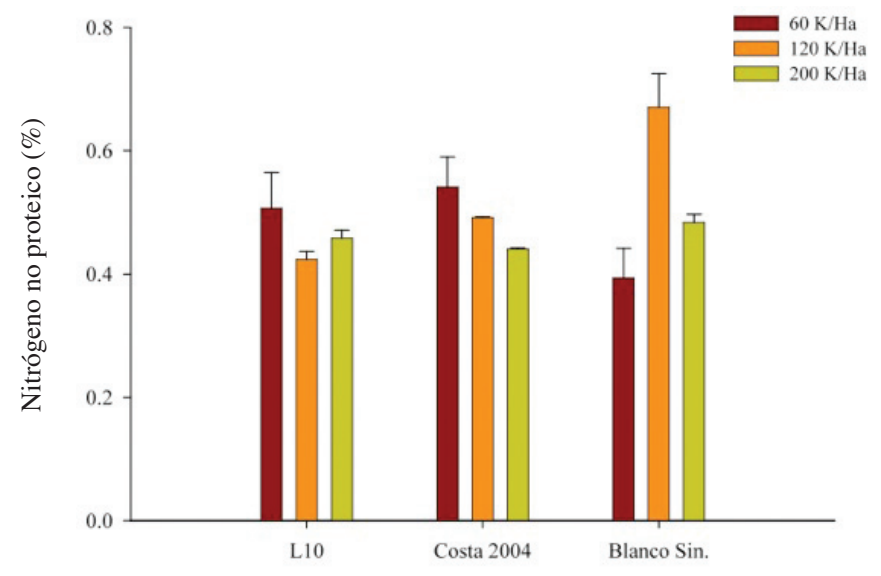

Variedades

Medias con diferente letra son significativamente diferentes $(\mathrm{p}<0.05)$.

\section{FIGURA 2}

Nitrógeno proteico presente en variedades de garbanzo sembradas a diferentes niveles de fertilización.

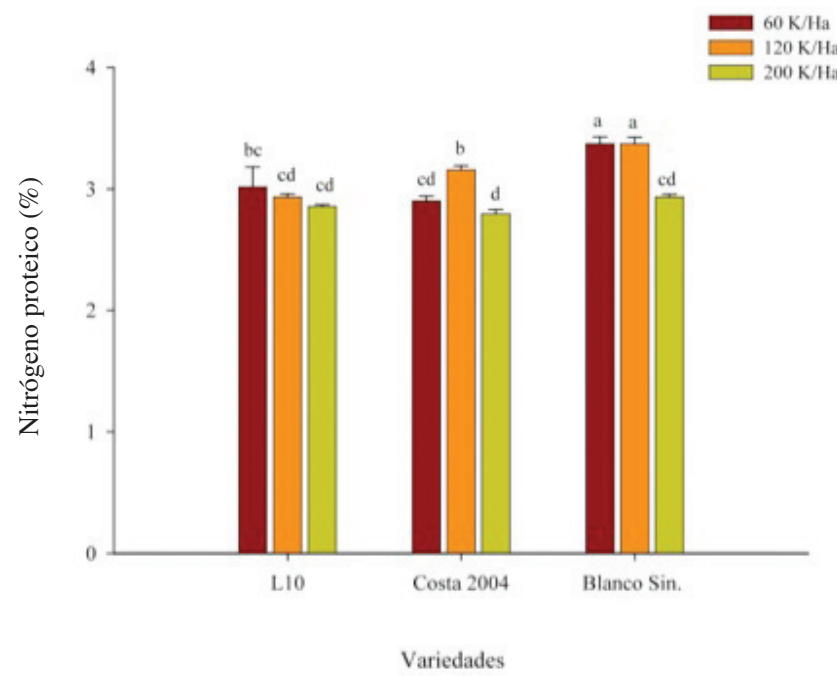

Medias con diferente letra son significativamente diferentes $(\mathrm{p}<0.05)$. 
fertilización de 200 mostraron los resultados más bajos en todos los casos. Las prácticas de manejo utilizadas por los productores de garbanzo tanto en la siembra como en su desarrollo tienen un efecto significativo en la calidad y cantidad de proteína (16).

Estudios realizados por Luo et al (17) en cultivo de trigo detectaron que la adición de nitrógeno después de la floración favorece tanto la cantidad como la composición de la proteína del grano. Thomason et al (18) y Rogers et al (19) reportaron que la absorción de nitrógeno por la planta de trigo, depende no sólo de la cantidad adicionada de $\mathrm{N}$, sino del balance que debe de existir del $\mathrm{N}$ y $\mathrm{S}$ presente en la planta, ya sea adicionado o presente en el suelo, así como del tipo de fertilizante utilizado.

Se puede observar que un nivel mayor de fertilización, no es determinante para obtener una mayor cantidad de nitrógeno proteico en el grano de garbanzo. Esto quiere decir que el nitrógeno añadido al terreno de siembra, no necesariamente es absorbido en su totalidad ó al ser absorbido por la planta, no precisamente se convertirá en nitrógeno proteico, ya que la planta lo puede utilizar en su desarrollo. Ha sido ampliamente documentado que el contenido de nitrógeno proteico en el arroz está influenciado no sólo por la cantidad de nitrógeno aplicado en el cultivo sino también por el tipo de fertilizante utilizado y que la cantidad de nitrógeno añadido al suelo afecta la cantidad de la fracción de glutenina (20).

\section{Efecto del grado de fertilización y variedad en el porcentaje de digestibilidad in vitro}

El contenido de aminoácidos es el principal indicador de la calidad de la proteína, pero la verdadera calidad depende de la utilización de los mismos por el organismo, es por esto que la digestibilidad es considerada como un indicador de la calidad de la proteína (21). En este estudio el porcentaje de digestibilidad in vitro fue utilizado como un indicador de la calidad proteica de garbanzo. En la Figura 3 se observan porcentajes de digestibilidad muy similares entre las muestras de garbanzo, indicando de esta manera que la variedad no tuvo efecto en la digestibilidad proteica in vitro. Sin embargo, la fertilización si afectó significativamente $(\mathrm{p}<0.05)$ la digestibilidad, siendo las muestras de garbanzo con fertilización de $200 \mathrm{~K} / \mathrm{Ha}$, las de menor digestibilidad.

Los resultados obtenidos en esta determinación abarcaron un rango de $72 \%$ a $79 \%$. El valor menor fue el obtenido de la variedad Costa con un nivel de fertilización de 200, mientras que el valor más alto fue para la variedad Blanco Sinaloa con un nivel de fertilización de 120. En la línea avanzada Hoga L10 no se encontraron diferencias significativas entre los tres niveles de fertilización: La variedad Costa con fertilización de $200 \mathrm{k}$ /ha fue la que presentó una menor digestibilidad, seguida del nivel 120 y de 60K/Ha; no encontrándose diferencias significativas entre los niveles de 60 y $120 \mathrm{~K} /$ Ha. En la variedad Blanco Sinaloa se observó que el nivel

\section{FIGURA 3}

Digestibilidad in vitro de variedades de garbanzo sembradas a diferentes niveles de fertilización.

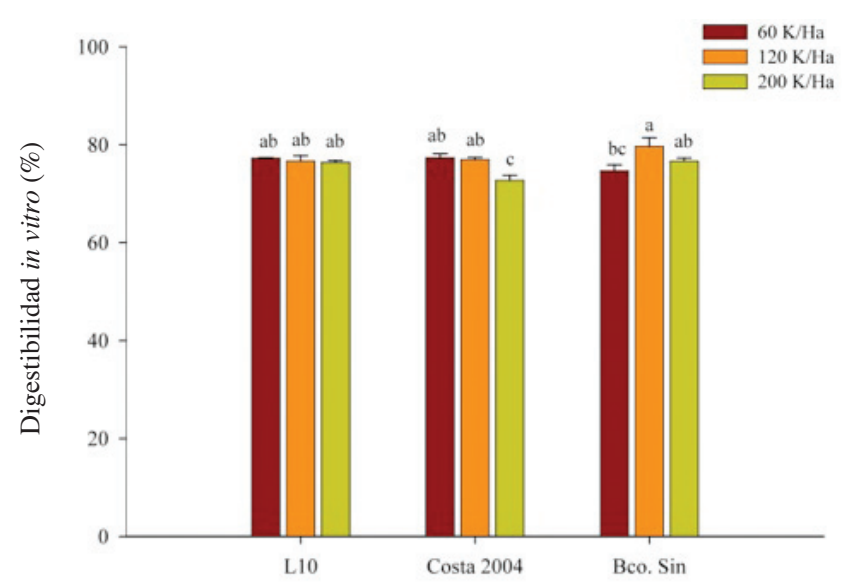

Variedades 
de fertilización que presentó una menor digestibilidad fue el de $60 \mathrm{~K} / \mathrm{Ha}$ y la mayor digestibilidad la presentó el garbanzo sometido a una fertilización de $200 \mathrm{~K} / \mathrm{Ha}$, siendo estas diferencias estadísticamente significativas $(\mathrm{p}<0.05)$.Estos valores de digestibilidad concuerdan con los encontrados en estudios realizados por Carbonaro et al (22), en los cuales se reportaron porcentajes de digestibilidad in vitro de $78.28 \%$ para garbanzo crudo. Así mismo Han et al (23) estimaron un $74.3 \%$ de digestibilidad para el garbanzo sin tratamiento. Sin embargo Pons et al (24) encontraron que los diferentes niveles de fertilización nitrogenada $(45$ y $90 \mathrm{~K} / \mathrm{Ha})$ no afectaron significativamente $(\mathrm{P}<0.05)$ la digestibilidad in vitro en maíz.

\section{Efecto del grado de variedad y fertilización en el contenido de lisina disponible}

Al hacer comparación de los resultados de lisina disponible entre las variedades (figura 4), Blanco Sinaloa fue la que registró los valores mas bajos, siendo Costa y la línea Hoga L10 las que mostraron los valores más altos, encontrándose estas diferencias estadísticamente significativas $(\mathrm{P}<0.05)$. Los resultados obtenidos en esta investigación fueron más altos que los reportados por Hurrel et al (11) en estudios anteriores. Las diferencias entre estos resultados y los obtenidos en esta investigación probablemente se deba al uso de otras variedades de garbanzo.
La fertilización afectó significativamente la disponibilidad de lisina. A excepción de Costa las variedades sembradas con nivel de fertilización de $200 \mathrm{~K} / \mathrm{Ha}$ fueron las que presentaron los valores más altos de lisina disponible. En la línea avanzada Hoga L10 no se encontraron diferencias significativas entre los tres niveles de fertilización. En la variedad Costa se puede observar que el garbanzo que mostró un mayor contenido de lisina disponible fue el que recibió un nivel de fertilización de 120 , seguido de 60 y 200K/Ha con los valores menores; no encontrándose diferencias significativas entre los niveles de 120 y 60 . En la misma Figura se puede observar que para la variedad Blanco Sinaloa el nivel que arrojó los valores mas altos de lisina disponible fue el de $200 \mathrm{~K} / \mathrm{Ha}$, siendo estadísticamente diferente con respecto a los otros niveles de fertilización (figura 4).

\section{CONCLUSIONES}

En general las variedades y línea avanzada de garbanzo estudiadas presentaron diferencias significativas $(\mathrm{p}<0.05)$ en el contenido de nitrógeno proteico, no proteico y lisina disponible. Ademas se encontró una influencia significativa de la fertilización en todas las características nutricionales evaluadas. El uso de niveles más altos de fertilizantes nitrogenados no necesariamente redunda en una mejor calidad proteica. En este estudio el nivel 120K/Ha mostró los resultados más aceptables para la mayoría de las variedades.

\section{FIGURA 4}

Lisina disponible de variedades de garbanzo sembradas a diferentes niveles de fertilización.

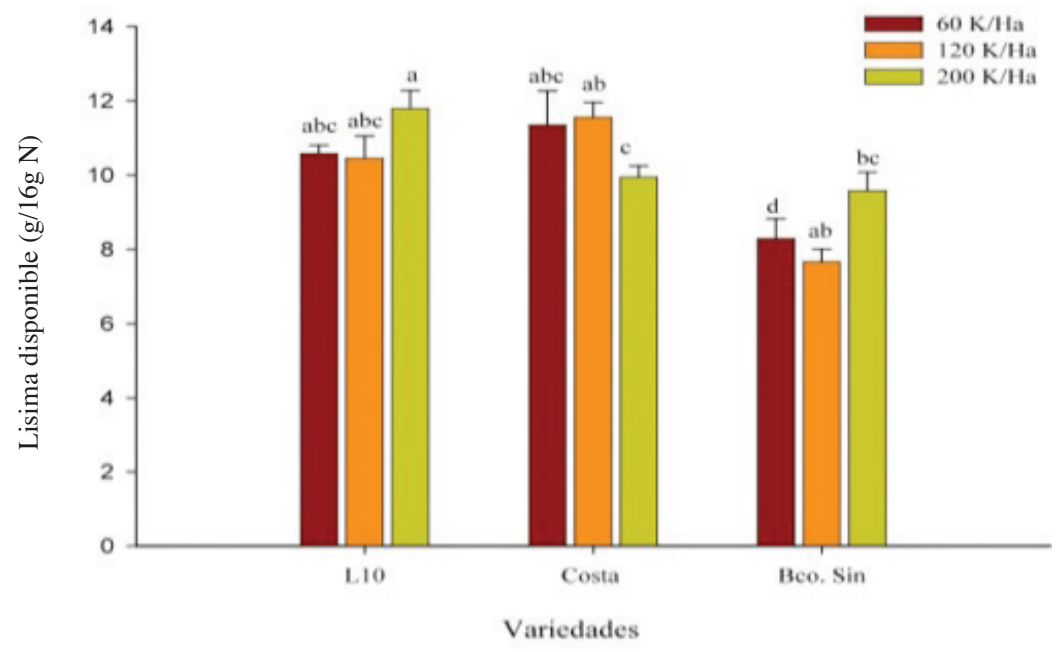

Medias con diferente letra son significativamente diferentes $(\mathrm{p}<0.05)$. 
Es importante resaltar que no necesariamente una variedad de mayor contenido de nitrógeno proteico, resulta en una mayor calidad proteica, ya que también depende de otros factores como la cantidad de aminoácidos esenciales que conforman la proteína y la disponibilidad de estos.

\section{RESUMEN}

En este trabajo se evaluó la calidad proteica in-vitro en dos variedades y una línea avanzada de garbanzo, así como su relación con el grado de fertilización aplicado durante su cultivo. Se utilizaron dos variedades comerciales de garbanzo (Costa y Blanco Sinaloa) y una línea avanzada Hoga L10, sembradas en condiciones ambientales similares, con tres niveles de fertilización de nitrógeno $(60,120$ y 200K/Ha.), las cuáles fueron obtenidas del Campo Experimental Costa de Hermosillo, Sonora, México del INIFAP. Se llevó a cabo un análisis químico proximal, determinaciones de calidad proteica como: nitrógeno proteico y no proteico utilizando extracciones con ácido tricloroacético, digestibilidad invitro por el método multienzimático y lisina disponible. A excepción de digestibilidad in-vitro se observaron diferencias significativas entre las variedades y línea avanzada en: nitrógeno proteico, no proteico y lisina disponible. El efecto de la fertilización fue observado en todos las determinaciones de calidad proteica, los resultados indicaron que al aumentar el nivel de fertilización a $120 \mathrm{~K} / \mathrm{Ha}$ se obtiene un incremento significativo en el porcentaje de nitrógeno proteico y no proteico, así como el porcentaje de digestibilidad. Entre los niveles de 60 y $200 \mathrm{~K} / \mathrm{Ha}$ no se observaron diferencias significativas. La lisina disponible fue mayor en las variedades y líneas con nivel de fertilización de 200K/Ha. Estos indicadores de calidad proteica pueden complementar a los aspectos agronómicos y comerciales que son evaluados en los programas de mejoramiento, en la selección de nuevas variedades de garbanzo para su explotación comercial.

Palabras clave: calidad proteica in-vitro; garbanzo; variedad; fertilización; nitrógeno.

Dirigir la correspondencia a:

Profesora

Alma Guadalupe Cota

Departamento de Investigación y Posgrado

en Alimentos.

Universidad de Sonora, México

Blvd. Encinas y Rosales s/n.

Col. Centro Hermosillo

Sonora, México

Apartado postal 1658

Teléfono: (662) 2592208
Fax: (662) 2592207

E-mail:agcota@guayacan.uson.mx

\section{BIBLIOGRAFÍA}

1. Marginet. JL. El garbanzo y sus perspectivas. Ambiente Ecológico. Multimedios Ambiente Ecológicg - MAE. 2008. ISSN 1668-3358, www.mae.org.an

2. Coskuner Y, Karababa E. Effect of Location and Soaking Treatments on the Cooking Quality of Some Chickpea Breeding Lines. International J Food Science Technol 2003;38:751-757.

3. Milán-Carrillo J, Valdéz-Alarcón C, GutiérrezDorado R, Cárdenas-Valenzuela, OG, MoraEscobedo R, Garzón-Tiznado JA, Reyes-Moreno C. Nutritional Properties of Quality Protein Maize and Chickpea Extruded Based Weaning Food. Plant Foods Human Nutr 2007; 62: 31-37.

4. SAGARPA,2007 Anسariosstadísticode la Producción Agrícola. www.siap.sagarpa.org.mx

5. INIFAP.2008. Informe Anual de Actividades del Instituto Nacional de Investigaciones Forestales, Agrícolas y Pecuarias http//wwww inifap.gob.mx/ temas_interes/informe_actividades.htm.

6. Ravi R, Harte JB. Milling and physicochemical properties of chickpea (Cicer arietinum L.) varieties. J Sci Food Agric 2009; 89: 258-266.

7. Morales GJA, Manjares SP, Salinas SP. Mejoramiento Genético y Variedades. Capitulo 4. El cultivo de Garbanzo Blanco en Sonora. Libro Técnico Núm. 6, INIFAP, Hermosillo, Sonora, México. 2004.

8. AACC. Approved Methods for Analysis American. American Association of Cereal Chemists. St Paul Minn 2001.

9. Singh U, Jambunathan R. Relationship Between Nonprotein and Total Nitrogen in Chickpea (Cicer arietinum) Seed. J Agriculture Food Chem 1981;29, 423-424.

10. Satterlee LD, Kendrick JG, Marshall HF, Jewell DK, Heckman MN, Steinke HF, Larson P, Phillips RD, Sarwar G, Slump P. In-Vitro Assay for Predicting protein Efficiency Ratio as Measured by Rat Bioassays: Collaborative Study. J Assoc Anal Chem 1982; 65: 798-809.

11. Hurrel RF, Lerman P, Carpenter KJ. Reactive Lysine in Foodstuffs As Measured by a Rapid Dye-Binding Procedure. J Food Science 1979; 44:12-22.

12. SAS. JMP Version 5.1. Statistics and Graphics Guide. SAS Institute Inc., Cary, NC, USA. 2002.

13. Vargas-Torres A, Osorio-Díaz P, Agama-Acevedo E, Morales-Franco L, Bello-Pérez L A. Starch digestibility in different bean (Phaseolus vulgaris 
L.) varieties. Interciencia 2006;31 (12): 881-884.

14. Perez HP, Esquivel EG, Rosales SR. Caracterización física, culinaria y nutricional de frijol del altiplano subhúmedo de México. ALAN 2002;52 (2):172-180.

15. Navarro AL. Caracterización Parcial de las Proteínas Mayoritarias de Garbanzo Nacional, de Exportación y Rezaga y su Posible Relación con Calidad Comercial. Tesis de Maestría. Depto. de Investigación y Posgrado en Alimentos (DIPA), Universidad de Sonora. Hermosillo, Sonora, México. 1997.

16. Khan MA, Akhtar N, Ullah I, Jaffery S. Nutritional evaluation of desi and kabuli chickpeas and their products commonly consumed in Pakistan. Int $\mathbf{J}$ Food Sci Nutr 1995; 46: 215-223.

17. Luo C, Branlard G, Griffin B, Mc Neil DL. The effect of nitrogen and sulphur fertilization and their interaction with genotype on wheat glutenins and quality parameters. J Cereal Sci 2000;31: 185-194.

18. Thomason WE, Phillips SB, Pridgen TH, Kenner JC, Gryffey CA, Beahm BR, Seabourn BW. Managing Nitrogen and sulfur fertilization for improved bread wheat quality in humid environment. Cereal Chem 2007; 84(5):450-462.

19. Rogers WJ, Cogliatti M, Lerner SE, Ponzio NR, Robutti JL, Di Martino AM, Borrás F, S, Seghez- zoand ML, Molfese ER. Effects of nitrogen and sulfur fertilizers on gliadin composition of several cultivars of durum wheat. Cereal Chem 2006; 83(6):677-683.

20. Champagne ET, Bett-Garber KL, Thomson JL, Fitzgerald MA. Unraveling the impact of nitrogen nutrition on cooked rice flavor and texture. Cereal Chem 2009; 86(3):274-280.

21. Damedaran S. Aminoacid, peptides and protein. Fennema O. R. 2008. Ch. 5. In Fennena's Food Chemistry. Damedaran S, Parkin KL, Fennema O.R. (Ed.). pp. 217-329. Fourth Edition. CRC press. New York. 2008.

22. Carbonaro M, Capelloni M, Nicoli S, Lucarini M, Carnovale E. Solubility-Digestibility Relationship of Legume Proteins. J Agric Food Chem 1997;45:3387-3394.

23. Han IH, Swanson BG, Baik B.K. Protein digestibility of selected legumes treated with ultrasound and high hydrostatic pressure during soaking. Cereal Chem 2007;84(5):518-521.

24. Pons CM, Arelovich HM, Amela MI, Mockel F. Productividad de Biomasa Forrajera de Dos Variedades de Maíz Bajo Riego y Valor Nutritivo del Silaje. Dto. Agronomía, Univ. Nac. del Sur. CIC Pcia. de Buenos Aires. 2002. 